\title{
LA CATEGORÍA DE LUGAR: DE LA GRAMÁTICA AL DISCURSO
}

\author{
(The category of Place: Grammar to Discourse)
}

\author{
María Laura Pardo ${ }^{1}$ \\ (CONICET-Universidad de Buenos Aires - UBA)
}

\begin{abstract}
The aim of this paper is to discuss some concepts related to synchro-diachronic method to the linguistic analysis of texts (from now on MSDALT) (PARDO, 1996, 2011). This is an exploratory research. Specifically we try to account for the grammaticalized category and, therefore, obligatory Place. It is recognized the orientative role of the category Place; however, this can change its role in the text and become a category of semantic-discursive character. In addition, this category becomes one of the topics of the text in which it is included. This re-functionality of the category of Place seems to keep a link with the postmodern discussion about space, and this happened in modernity with grammaticalized Time category. This topic is outlined in this paper for future research. The theoretical framework of this research is Discourse Analysis. The methodology is qualitative and the selected corpus is made up of news on YouTube chronic surveyed, of which two are taken as examples. The linguistic analysis is performed by the aforementioned method (PARDO, 2011).
\end{abstract}

Key words: Place, Grammar, Discourse, MSDALT

\section{RESUMEN}

El objetivo de este trabajo es discutir algunas nociones relacionadas con el Método sincrónico-diacrónico de análisis lingüístico de textos (de aquí en más MSDALT), (PARDO, 1996, 2011). Se trata de una investigación exploratoria. Específicamente intentaremos dar cuenta de la categoría gramaticalizada y, por lo tanto, obligatoria de Lugar. Es reconocida la función orientadora de la categoría Lugar; sin embargo, esta puede cambiar su función en el texto y constituirse en una categoría de carácter semánticodiscursivo. Además, dicha categoría se torna uno de los tópicos del texto en que se incluye. Esta refuncionalidad de la categoría de Lugar parecería guardar un vínculo con la discusión posmoderna acerca del espacio, así como en la modernidad esto sucedía con la categoría gramaticalizada de Tiempo. Este tema queda esbozado en este trabajo para investigaciones futuras. El marco teórico de esta investigación es el Análisis del Discurso. La metodología es cualitativa y el corpus seleccionado está conformado por crónicas noticiosas relevadas en YouTube, de las cuales se toman dos a modo de ejemplos. El análisis lingüístico se realiza mediante el método antes mencionado (PARDO, 2011).

Palabras clave: Lugar, gramática, discurso, MSDALT

\footnotetext{
${ }^{1}$ La autora es Dra. en Letras por la Facultad de Filosofía y Letras (FFYL) de la Universidad de Buenos Aires (UBA), investigadora independiente del Consejo Nacional de Investigaciones Científicas y Técnicas (CONICET). Directora del Departamento de Lingüística del Centro de Investigaciones en Antropología Filosófica y Cultural (CIAFIC)-CONICET. Profesora de Análisis de los lenguajes de los medios masivos de comunicación social en la FFYL de la UBA. Vicepresidente de la Asociación Latinoamericana de Estudios del Discurso (ALED). 


\section{Introducción}

La idea de Lugar en la gramática siempre ha sido vista como lo que se denomina un complemento y, además, circunstancial. Es circunstancial en tanto señala las circunstancias en las que se da el hecho que se narra. El Complemento Circunstancial de Lugar tiene la función sintáctica de informar el lugar en el que transcurre la acción del verbo. Suele responder a la pregunta ¿Dónde?

Los complementos circunstanciales expresan cualquier circunstancia de la acción significada por el verbo; tal como tiempo, lugar, modo, materia, instrumento o compañía. Son por naturaleza complementos circunstanciales todos los adverbios. Los formados por varias palabras llevan siempre preposición; cuál sea ésta depende no sólo de la circunstancia que se expresa sino del verbo o de la palabra afectada por la preposición. Como no es posible dar reglas completas, en los artículos correspondientes a los nombres o verbos sobre cuyo régimen puede caber duda, se consignan las preposiciones con que se les unen sus complementos (MOLINER, 1998 [1966-1967])

El complemento circunstancial ha sido siempre una categoría difícil de definir en las gramáticas. Sin embargo, tres teorías postulan un tratamiento bastante similar para para dichos complementos, conforme Celia Berná Sicilia (2013):

A pesar de la distancia teórica y metodológica que los separa, estos tres planteamientos (la Teoría de la Valencia (Tesnière, 1994), el Funcionalismo (Dik, 1981) o el Generativismo, sobre todo a partir de las formulaciones de los años 80 (Chomsky, 1981) coinciden en señalar la necesidad de diferenciar, dentro del entorno de la predicación verbal, dos tipos esenciales de componentes sintácticos: a) actantes (Tesnière, 1994) o argumentos (Dik, 1981) y b) circunstantes (Tesnière, 1994), satélites (Dik, 1981) o adjuntos. Los primeros son elementos predicacionales nucleares o centrales para identificar el estado de cosas denotado por el verbo y se caracterizan por ser requeridos específicamente por el núcleo verbal del que dependen (están semánticamente seleccionados o subcategorizados); los segundos, por el contrario, se configuran como complementos adicionales no seleccionados o no exigidos y poseen un carácter marginal, por lo que resultan generalmente optativos. (BERNÁ SICILIA, 2013, p. 4)

Esta investigación no apunta a mejorar la clasificación de los complementos circunstanciales en la gramática, que aún se torna compleja y rica a la vez, sino que desde otra perspectiva busca ahondar en la categoría de Lugar desde un vínculo más cercano a la función semántica que esta pueda tener en los textos o discursos. Desde otra mirada, un poco más amplia y en relación con las narrarivas, para Labov y Waletzky (1967), así como para Labov en trabajos 
posteriores (LABOV, 2010, p. 2), en las narrativas de experiencias personales, el tiempo, el lugar, y la conducta inicial son parte de la orientación" : "a narrative normally begins with an Orientation, introducing and identifying the participants in the action: the time, the place, and the initial behavior. The orientation section provides answers to the potential questions, "who? when? where? what were they doing?" ?

Resulta que esta función orientadora se refuncionaliza en algunos textos donde, si bien al comienzo puede ser orientadora, luego se refuncionaliza dando pié a un tópico textual. Es en este caso, cuando la categoría Lugar deja de ser puramente una categoría gramaticalizada para tornarse semántico-discursiva.

Cabe observar que Labov señala la función orientadora de Lugar para las narrativas sobre experiencias personales. Sin embargo, él mismo reconoce que los sectores que postuló con Waletzky en las narrativas: orientación, nudo, descomplejización, coda y evaluación también pueden darse en otras narrativas de muy variado tipo:

Labov and Waletzky demonstrated that the effort to understand narrative is amenable to a formal framework, particularly in the basic definition of narrative as the choice of a specific linguistic technique to report past events. The L\&W framework developed for oral narratives of personal experience proved to be useful in approaching a wide variety of narrative situations and types, including oral memoirs, traditional folk tales, avant garde novels, therapeutic interviews and most importantly, the banal narratives of every-day life. It allowed us to understand pseudo-narratives like recipes, apartment-house layouts, and other types of experience remodeled into narrative form. It gradually appeared that narratives are privileged forms of discourse which play a central role in almost every conversation. Our efforts to define other speech events with comparable precision have shown us that narrative is the prototype, perhaps the only example of a well formed speech event with a beginning, a middle, and an end $(\mathrm{LABOV}, 2010)^{4}$

${ }^{2}$ Es importante aclarar que, si bien Labov trabaja con cláusulas, estas nociones ligadas a la orientación pueden extenderse a la emisión y por lo tanto al texto y es en este sentido que son relevadas en este trabajo.

${ }^{3}$ Traducción al español: "Una narrativa, normalmente, comienza con una Orientación, introduciendo e identificando a los participantes en la acción: el tiempo, el lugar, y la conducta inicial. La sección de la orientación provee las respuestas a preguntas potenciales como: ¿quién?, ¿cuándo?, ¿dónde?, ¿qué estaban haciendo?” (LABOV, 2010, p. 2).

${ }^{4}$ Traducción al español: Labov y Waletzky demostraron que el esfuerzo para entender una narrativa es susceptible a un marco formal, particularmente en la definición básica de la narrativa como la elección de una técnica lingüística específica para informar sobre eventos pasados. El marco teórico desarrollado por L\&W a partir de narraciones orales de experiencia personal ha demostrado ser útil para acercarse a una amplia variedad de situaciones y tipos narrativos, incluyendo memorias orales, cuentos populares tradicionales, novelas vanguardistas, entrevistas terapéuticas y lo más importante, los relatos banales de la vida cotidiana. Nos permitió entender pseudo-narrativas como las recetas, diseños de apartamento-casa y otros tipos de experiencia remodelados en forma narrativa. Al 
Ahora bien, la teoría que se propone en el MSDALT sostiene que las narraciones suelen ser modos de dar evidencia dentro de textos que tienen una función más argumentacional, entendiendo por argumentacional una dimensión del lenguaje que es gradual y va de un grado mínimo a uno máximo de argumentatividad, llegando en el lado máximo de la escala a constituirse un género o práctica discursiva que podemos llamar argumentación o retórica. ${ }^{5}$ De este modo, las narrativas se presentan como estrategias discursivas que están al servicio de las tesis que se sostienen en un texto, dando evidencia, mediante experiencias propias o ajenas, de que lo que se afirma es cierto.

En esta investigación intentaremos dar cuenta de la categoría Lugar, que puede o no tener en el inicio del texto una función orientadora, pero que a medida que el texto avanza va enriqueciéndose semánticamente hasta constituirse en uno de los tópicos más relevantes del texto.

\section{Presentación del Método sincrónico-diacrónico de análisis linguiístico de textos}

El método sincrónico-diacrónico de análisis lingüístico de textos constituye, por un lado, una teoría acerca del lenguaje y, por otro, un método de análisis lingüístico de textos. Además, dado que es un método inductivo que relaciona representaciones discursivas con representaciones sociales funciona como una teoría básica que permite unir la práctica textual con la social (en términos de Fairclough, 1995) mediante datos nacidos en el análisis (Strauss y Corbin, 2002).

En el MSDALT se distinguen dos tipos de categorías, las gramaticalizadas y las semántico-discursivas. Estas pueden seguirse en la sincronía de la emisión o en la diacronía del texto. La teoría se basa en varios principios del lenguaje: la jerarquización de la información, el dialogismo, la tonalización y la argumentación. Los menciono como principios pues el lenguaje es dialógico por naturaleza. El hablante dialoga interna y/o externamente con ese "otro" social, lo que hace que se requiera de una argumentación, tiene la pretensión (consciente o inconsciente) de, al menos, alcanzar el "entendimiento" (Habermas, 1986) del otro, de igual manera jerarquiza

parecer, poco a poco, las narrativas son formas privilegiadas de discurso que desempeñan un papel central en casi todas las conversaciones. Nuestros esfuerzos para definir otros hechos de habla con una precisión comparable nos han demostrado que la narrativa es el prototipo, tal vez el único ejemplo de un hecho de habla bien formado con un principio, un medio y un final. (Labov, 2010).

${ }^{5} \mathrm{La}$ argumentatividad es el grado de dinamismo comunicativo con que el texto avanza hasta alcanzar aquello que el hablante se proponía decir o sostener específicamente en términos de argumentos. 
la información (entendiendo por jerarquización en el plano macro: el texto) y la tonalización (como una jerarquía en el plano micro, es decir, la emisión) ${ }^{6}$.

Todos los textos orales y escritos tienen un número de categorías gramaticalizadas y que, en general, son de carácter obligatorio, independientemente del género discursivo (Bajtín, 1988) que se maneje. Ellas son:

- Hablante-Protagonista (H-P de aquí en más): este se corresponde con cualquier persona pronominal o cualquier referente nominal que tome el argumento del hablante. Es importante aclarar que esta categoría no necesariamente aparece en la posición de sujeto gramatical o lógico de la emisión;

- Verbo 1 (o Nexo de Valor 1) con el que el hablante actúa de alguna manera. Este verbo señala solo las acciones del sujeto y no la de otros actores en el texto;

- Actor/Actores: cualquier persona pronominal o referente nominal que toma los argumentos opuestos a los que sostiene el hablante;

- Verbo 2 (Nexo de Valor 2) con el que el Actor/Actores accionan,

- Tiempo;

- Lugar;

Tanto la categoría de Tiempo como la de Lugar responden a la orientación espaciotemporal necesaria y obligatoria de cualquier texto.

- Operador pragmático: suele aparecer en todos los textos y tiene distintas funciones, desde la de señalar cómo debe interpretarse una parte de la emisión, la de conectar distintas emisiones o sectores en la emisión o como recurso para interpelar o lograr la complicidad del oyente o lector,

- Negación: esta categoría es lo que denomino una categoría flotante, ya que puede aparecer negando al verbo, a otras palabras o a sectores de una emisión. Esta categoría no tiene el mismo grado de obligatoriedad que las restantes.

Por otra parte, tenemos las categorías semántico-discursivas o no gramaticalizadas. Estas son de carácter no obligatorio ya que varían de texto a texto, dependen además de la

\footnotetext{
${ }^{6}$ Otras nociones propias del método como las de Nexo de Valor o la teoría de los desplazamientos pueden verse en Pardo (2011). 
interpretación del investigador. Además, para ser consideradas una categoría de este tipo, en las emisiones restantes del texto debe haber referencias directas o indirectas al tema de la categoría en cuestión. Cada categoría semántico-discursiva puede ser analizada como una unidad y estudiada en profundidad en la observación sincrónica (a lo largo de la emisión) y en la diacrónica (a lo largo del texto), pero también podemos verlas, como propiedades, esto es, como categorías semántico-discursivas de una representación discursiva (y, por lo tanto, social) macro. Lo mismo sucede con las categorías gramaticalizadas. A continuación damos inicio al análisis lingüístico mediante el MSDALT.

\section{La categoría gramaticalizada Lugar con función orientadora}

\section{Evento 1: Crónica televisiva de Todo Noticias ${ }^{7}$}

Periodista: 1[Vamos a ver un robo, vamos a ver el momento en que se produce ese robo, es el robo de un estéreo, es un auto, esto es en la zona de la Facultad de Medicina y ese chico es el ladrón.] 2[Acaba de abrir la puerta, con una yuga, con una especie de una punta de tijera para que se hagan la idea.] 3[Activó la alarma y el estéreo en el pantalón, se tapa con la remera, tiene una remera ancha.]

\footnotetext{
${ }^{7}$ El Evento 1 forma parte de un corpus de 10 crónicas televisivas de diferentes canales de aire y cable reproducidas en YouTube acerca de la problemática que vincula a los jóvenes que viven en situación de pobreza, con el delito y con la villa. (https://www.youtube.com/watch?v=2mqCurcFuKc).
} 
Cuadro 1: Aplicación del MSDALT al Evento 1

\begin{tabular}{|c|c|c|c|c|c|c|c|}
\hline V1 & Delito & Tiempo & V2 & Auto & Lugar & Herramienta & Ropa \\
\hline $\begin{array}{l}\text { e1[Vamos a ver } \\
\text { vamos a ver } \\
\\
\text { para que se } \\
\text { hagan }\end{array}$ & $\begin{array}{l}\text { un robo, } \\
\text { en que se } \\
\text { el robo } \\
\text { esto } \\
\text { y ese chico } \\
\text { el ladrón] }\end{array}$ & el momento & \begin{tabular}{|l} 
produce \\
es \\
es \\
\\
es \\
2[Acaba \\
de abrir \\
\\
\\
3[Activó \\
y mira \\
se guarda \\
se tapa \\
tiene
\end{tabular} & $\begin{array}{l}\text { de un } \\
\text { estéreo } \\
\text { un auto }\end{array}$ & $\begin{array}{l}\text { en la zona } \\
\text { de la Facultad } \\
\text { de Medicina }\end{array}$ & \begin{tabular}{|l} 
\\
con una yuga \\
con una especie \\
de una punta \\
de tijera
\end{tabular} & \begin{tabular}{|l} 
\\
\\
ahí \\
en el pantalón \\
con la remera \\
una remera \\
ancha]
\end{tabular} \\
\hline
\end{tabular}

Luego de aplicar el MSDALT podemos observar las categorías gramaticalizadas y las semántico-discursivas de este breve texto. Las gramaticalizadas son V1, Lugar, Tiempo, las semántico-discursivas son Delito, Auto, Herramienta, Ropa. La categoría de Hablanteprotagonista se encuentra en la desinencia verbal y es un "nosotros" inclusivo.

La categoría de Lugar tiene en este texto dos interpretaciones posibles. La primera posibilidad es que solo tiene una ocurrencia, tal como se la observa en el cuadro de aplicación de MSDALT: “en la zona de la Facultad de Medicina”. La aparición del "ahí", en esta apreciación, no refiere a una zona sino a un lugar en su ropa, el bolsillo, pero dado que la ropa se realiza luego, no solo con el bolsillo del pantalón sino con la remera que lleva puesta, se separa de la idea de lugar físico de ocurrencia del delito para indicar las características del ladrón. En este caso, la categoría de Lugar actúa como orientadora para el televidente, indicando el lugar del delito. En la segunda posibilidad, el "ahí" puede interpretarse como otra ocurrencia de la categoría de Lugar que estaría señalando el espacio físico en el que este Actor (el ladrón) guarda el estéreo, resignificando la zona del delito con la zona en la cual, en su propio cuerpo, guarda lo robado. De este modo, tenemos dos lugares, uno externo, la Facultad y otro interno, el cuerpo. Ambas con función orientadora. Responderían a dos preguntas sobre el dónde, ¿dónde fue el 
delito? ¿Y dónde se guardó el objeto del delito? En ninguno de los dos casos, el lugar, ya sea como complemento del verbo cópula o como adverbio de lugar son obligatorios, pero sí ambos responden a las características de los circunstanciales: "en la zona de la Facultad de Medicina" comienza con preposición (nexo subordinante) y sigue con el término cuyo núcleo es "zona", mientras que el "ahí", como dijéramos, es un adverbio de lugar.

\section{La categoría gramaticalizada de Lugar como una categoría a la vez semántico-discursiva}

Evento 2: Una crónica televisiva del canal de aire y cable América ${ }^{8}$

Cuadro 2: Aplicación del MSDALT al Evento 2

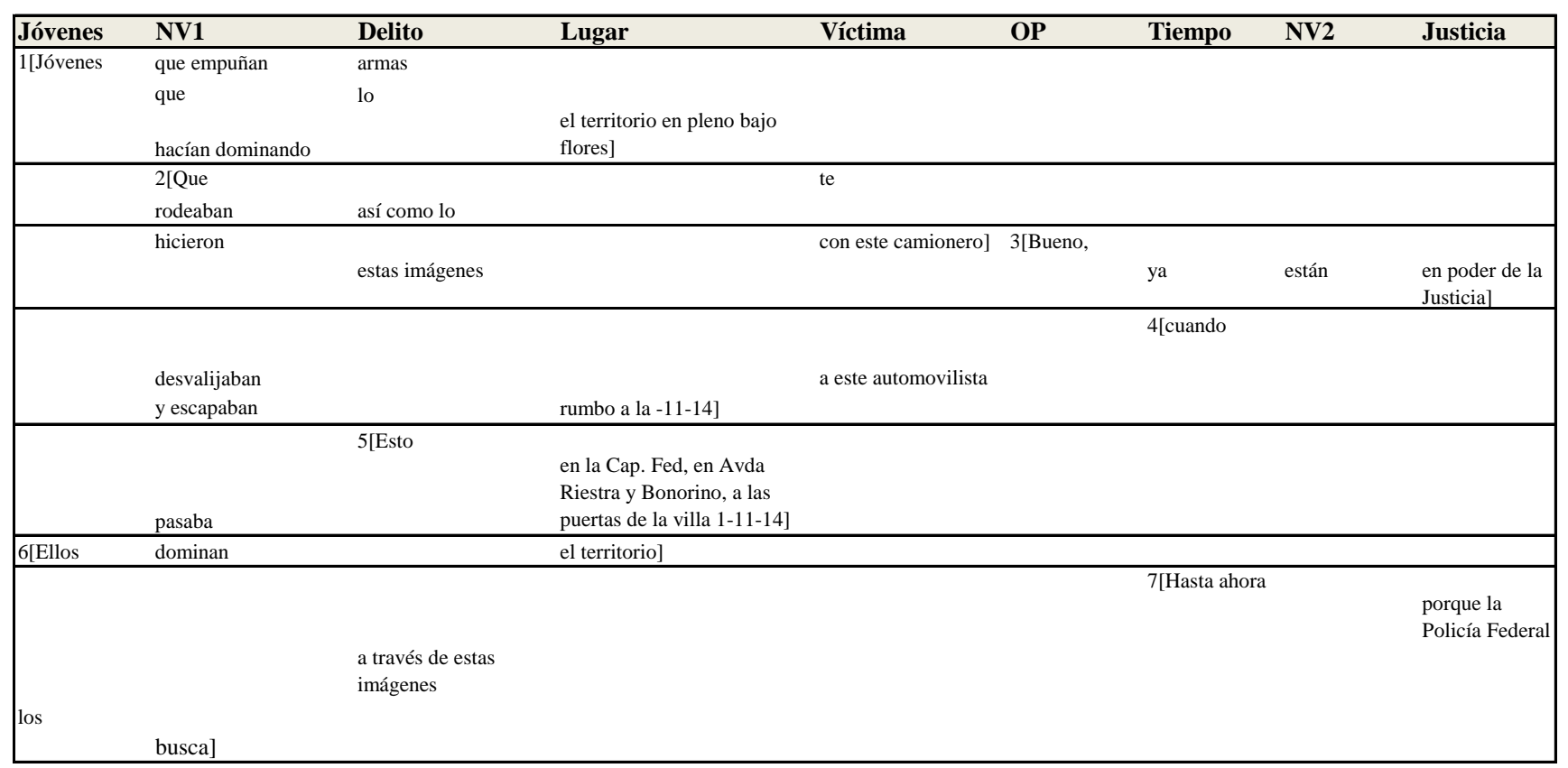

Si aplicamos el MSDALT, podemos observar las siguientes categorías gramaticalizadas: Verbos (V1), Tiempo, Lugar, Víctima que se equipara a la de Actores y Jóvenes que podría considerare un Hablante-Protagonista, en calidad no de Hablante pero sí de Protagonista de lo que se cuenta en la crónica, y Operador pragmático. Tenemos luego sola una categoría semántico-discursiva: Justicia.

\footnotetext{
${ }^{8}$ Para un análisis crítico del discurso de este ejemplo, véase Pardo, 2014. http://www.youtube.com/watch?v=5gPIV74zhzc 2:06'
} 
Sin embargo, la categoría gramaticalizada de Lugar se torna una categoría semánticodiscursiva al tornarse uno de los tópicos fundamentales del texto (la villa).

Cuadro 3: Focos del ejemplo 2 de la Categoría de Lugar

\begin{tabular}{|l|}
\hline Focos de la categoría de Lugar \\
\hline $1[\ldots$ El territorio en pleno bajo flores $]$ \\
\hline $4[\ldots$ rumbo a la $11-14]$ \\
\hline $5[\ldots$ puertas de la villa $1-11-14]$ \\
\hline $6[\ldots$ el territorio $]$ \\
\hline
\end{tabular}

Si en el Cuadro 3 observamos diacrónicamente esta categoría, la primera ocurrencia es "el territorio en Bajo Flores". Si bien, el verbo que la antecede es "dominar" que es un verbo transitivo no rige que su Objeto Directo o Frase Nominal sea, necesariamente, un lugar o espacio (podría haber sido: "dominar el toro"; "dominar una lengua", que no son espacios). La segunda instancia de la categoría Lugar es "rumbo a la 11-14" y el verbo que la precede, en cambio, sí rige un complemento de Lugar: "escapaban". La tercera aparición es "en la Capital Federal, en Avda. Riestra y Bonorino, a las puertas de la villa 1-11-14". El verbo es "pasaba" que no rige una categoría de Lugar, podría haber sido de Tiempo o Modo ("pasaba la tarde con su abuelo"; “pasaba así mirando las estrellas”).

Por estos motivos, estos pasajes no están semánticamente seleccionados por los verbos. En esta ocasión (la tercera aparición), como vimos, la ocurrencia comienza con "en” (La Capital Federal), para seguir con otro "en” (Avenida Riestra y Bonorino”), para culminar esta cadena de espacios con la preposición o nexo subordinante "a" (a las puertas de la villa 1-1-14). Esta cadena de espacios, es importante notar, va haciendo cada más específico el lugar: va de la Capital Federal, pasando por las Avenidas, hasta llegar a la villa. La instancia que sigue de la categoría Lugar se encuentra en la emisión 6: "el territorio". Nuevamente con el verbo transitivo: "dominan".

Si analizamos esta categoría de Lugar en relación con todo el texto y, especialmente, con la categoría de Actor realizada como Jóvenes, nos encontramos con que estos son vinculados con dicha categoría a partir de que huyen hacia la Villa 1-11-14, "rumbo a la 11-14"; "en la Capital Federal, en Avda. Riestra y Bonorino, a las puertas de la villa 1-11-14”. También la villa es nominada aquí dos veces como "el territorio" (e1 y e6). Las acciones de los jóvenes representadas en NV1 son violentas: "empuñan armas"; "que te rodeaban"; "desvalijaban y 44 
escapaban"; y dos veces refiere el relato a que "dominan" el territorio (e1 y e6). De este modo, el término "territorio" aparece ligado a la 'dominación', como a la delimitación y mando que ejercen los animales frente a lo que creen propio. El verbo "Dominar" no exige una subcategorización semántica.

Ya el nombre "Territorio", por otra parte, es necesario en tanto el verbo, como dijimos, es transitivo, y no es suprimible ya que la emisión quedaría incompleta sin él. Es evidente que, en este caso, la categoría de Lugar no es orientadora para el oyente-lector sino que el espacio es algo que sirve a la argumentación del periodista que sostiene que estos jóvenes pelean por dicha zona para delinquir. En tanto, el término “1-11-14” está ligado a las acciones delictivas de los jóvenes.

Ahora bien, todos estos términos aparecen en foco. El término "foco" refiere a la Jerarquización de la Información (Pardo, 1996; Pardo, 2011), por la cual cada emisión puede entenderse con una parte temática (la que lleva la información que es de conocimiento compartido entre Hablante y Oyente; o Escritor-Lector), otra remática (que lleva la información nueva) y por último y dentro de la zona remática, la zona de foco, que coincide con la última parte de la emisión, en general, precedida por Frase Preposicional, "y" u "o”, o algún signo suprasegmental como "coma", "punto y coma", etc. El foco se da al final de las emisiones en las lenguas romances porque sigue el orden de palabras, en el caso del español: VSO.

En foco se encuentran todas las emisiones, dejando en evidencia que esto es lo que quien relata la nota quiere resaltar (véase cuadro debajo del MSDALT del ejemplo 2). Solo la e2, focaliza en una de las víctimas: el camionero y la e7 en que son buscados por la Policía, aunque esta última nunca está en foco. Lo que intenta mostrar la focalización es el vínculo joven-villadelito, en tanto en dos instancias a modo de círculo que se cierra aparece la víctima, que certifica el delito al igual que el hecho de que son buscados por la policía. De este modo, si bien puede decirse que la categoría de Lugar es orientadora para saber dónde se realiza el delito, la insistencia sobre ese espacio ("territorio") redimensiona la categoría de Lugar dándole una función que va más allá de ubicar al televidente en el lugar del delito, intenta también hacer hincapié en que estos jóvenes provienen de la villa y la dominan, transformándola en el espacio del delito. El lugar, de este modo, se transforma en eje de discusión, en sitio de estigmatización. 


\section{A modo de síntesis}

Muchos son los textos en los que la categoría de Lugar cobra una función que va más allá de ser orientadora, de este modo los circunstanciales de lugar pueden extender su significado y ser algo más que anexos del verbo.

En cuanto al género crónica es importante notar que, tal como su nombre lo indica, en las crónicas es fundamental el tiempo y otras formas de orientación. Sin embargo, en estas crónicas televisivas, especialmente las que tienen como temática los jóvenes que delinquen, la categoría de Lugar suele aparecer también como semántico-discursiva. La refuncionalización de una categoría no bloquea su función gramatical sino que la enriquece potenciando su significado.

A modo de comentario, y para profundizar en otras investigaciones, esta refuncionalización de la Categoría de Lugar, podría estar ligada a la explotación que se hace del espacio en la posmodernidad, especialmente de los llamados "no lugares" (Augé, 2000), tal como podría suponerse podrían ser los asentamientos en relación con el (mal) trato que suelen darle algunos vecinos y algunos funcionarios públicos.

Recebido em: junho de 2015 Autora convidada para este volume

pardo.linguistica@gmail.com

\section{Referencias bibliográficas}

AUGÉ, M. Los no lugares; espacios del anonimato. Barcelona: Gedisa, 2000.

BERNÁ S. C. Categorización moderna de los complementos circunstanciales: evolución de la tradición hispánica y revisión crítica. Tonos digitales, No. 25, Julio 2013.

CHOMSKY, N.. Lectures on government and binding. Dordrecht: Foris, 1981.

DIK, S. Gramática funcional. Madrid: Sociedad General Española de Librería, 1981.

FAIRCLOUGH, N. Critical discourse analysis: The critical study of language. London: Longman, 1995.

HABERMAS, J. La teoría de la acción comunicativa. Madrid: Taurus, 1986. 
LABOV, W. y WALETZKY, J. Narrative analysis. En HELM, J. (ed.) Essays on the verbal and visual arts. Seattle: University of Washington Press, p. 12-44, 1967. Reimpreso en el Journal of Narrative and Life History, vol.7, p.3-38, 1997.

LABOV, W. Some further steps in narrative analysis. En The Journal of Narrative and Life History, special issue, 1997.

. En http://www.ling.upenn.edu/ wlabov/sfs.html

- Narratives of personal experience. En HOGAN, P. (ed.) Cambridge encyclopedia of the language sciences. Cambridge: Cambridge University Press, 2010.

. http://www.ling.upenn.edu/ wlabov/Papers/FebOralNarPE.pdf

MOLINER, M. Diccionario de uso del español [CD-ROM]. Madrid: Gredos, 1998 [1966-1967].

PARDO, M.L. Derecho y Lingüística. Cómo se juzga con palabras. Buenos Aires: Nueva Visión, 1996a.

. Derecho y Lingüística. Cómo se juzga con palabras. Buenos Aires: Nueva Visión, $1996 b$.

- Teoría y metodología de la investigación lingüística. Método sincrónico-diacrónico de análisis lingüístico de textos. Buenos Aires: Tersites, 2011.

. Las representaciones socio-discursivas que sobre los jóvenes pobres ligados al delito crean los medios televisivos en Argentina. En: Romanica Olomucensia, Praga, República Checa, pp. $249-264,2014$.

STRAUSS, A. y J. CORBIN. Bases de la investigación cualitativa. Técnicas y procedimientos para desarrollar teoría fundamentada. Antioquia: Editorial Universidad de Antioquia.

TESNIÈRE, L. Elementos de sintaxis estructural. Madrid: Gredos, 1994. 